\title{
Cricothyroidotomy in the emergency setting: indications, techniques and outcomes
}

\author{
Federico Mazza ${ }^{1}$, Massimiliano Venturino ${ }^{1}$, Davide Turello ${ }^{1}$, Alberto Gorla ${ }^{1}$, \\ Cristina Degiovanni ${ }^{2}$, Alessandro Locatelli ${ }^{2}$, Giulio Melloni ${ }^{1, *}$
}

\author{
${ }^{1}$ Department of Thoracic Surgery, A.O. S. \\ Croce e Carle, 12100 Cuneo, Italy \\ ${ }^{2}$ Department of Anaesthesia and \\ Intensive Care, A.O. S. Croce e Carle, \\ 12100 Cuneo, Italy \\ *Correspondence \\ melloni.g@ospedale.cuneo.it \\ (Giulio Melloni)
}

\begin{abstract}
Cricothyroidotomy is a lifesaving procedure performed in acute respiratory failure secondary to upper airway obstruction to restore a patent airway. Emergency cricothyroidotomy is considered to be the technique of choice to provide oxygenation to patients in "Can't Intubate, Can't Oxygenate" (CICO) scenario. In this study, we describe and discuss indications, different techniques and complications of cricothyroidotomy performed for establishing an airway in the emergency setting from a multidisciplinary point of view.
\end{abstract}

\section{Keywords}

Cricothyroidotomy; Emergency; Airway; CICO
Cricothyroidotomy (also called cricothyrotomy) is a procedure that involves the creation of a communication between the skin and the airway through an incision of the cricothyroid membrane. It allows the placement of a small breathing tube into the trachea to establish a patent airway during certain life-threatening situations or to perform pulmonary toilette in patients unable to clear copious secretions.

Originally developed in the early $19^{\text {th }}$ century by the French surgeon Felix Vicq-d'Azyr, cricothyroidotomy was re-introduced 100 years later by the American laryngologist Chevalier-Jackson who described a technique, called "high tracheostomy", for gaining access to the trachea that resembles anything we see today [1-3]. Chevalier-Jackson's procedure used for prolonged mechanical ventilation was not welcomed in medical community and raised criticism because of high complication rates (in particular laryngeal stenosis) [4]. Because of these complications, cricothyroidotomy fell into oblivion until the 1970s when Brantigan et al. [3] demonstrated that, with some precautionary measures, this procedure can be performed safely and with few complications [5]. Since then cricothyroidotomy has been increasingly used in the clinical practice, not only for prolonged mechanical ventilation, but also as an emergency lifesaving maneuver to establish an airway for ventilation and oxygenation.

Nowadays, emergency cricothyroidotomy is universally considered as the technique of choice to provide oxygenation to patients in "Can't Intubate, Can't Oxygenate" (CICO) scenario [1, 6, 7] while elective cricothyroidotomy for prolonged mechanical ventilation has been definitively substituted by formal tracheostomy [8]. On the contrary, elective cricothyroidotomy, performed with prepacked kits (Mini-tracheostomy kit), continues to be used in the clinical practice in selected patients requiring frequent suctioning of secretions.

We describe and discuss indications, different techniques and complications of cricothyroidotomy performed for establishing an airway in the emergency setting from a multidisciplinary point of view, with particular emphasis on the clinical use of this potentially lifesaving procedure within the published guidelines for the management of difficult airway.

\section{Indications}

The primary indication for an emergency cricothyroidotomy is represented by a CICO scenario. CICO scenario is defined as a clinical situation characterized by the failure to intubate and oxygenate the patient as a result of an upper airway obstruction (at or above the subglottic region) [9]. As soon as the inability to intubate and oxygenate the patient is recognized, cricothyroidotomy should be performed to restore a patent airway, since continuing to attempt intubation increases morbidity and mortality [10-13]. The management of CICO scenario involves prompt decision-making to access the trachea via the cricothyroid membrane and it is described [1] as emergency front of neck access (eFONA).

There are several situations in which a physician may encounter a CICO scenario with the need to perform an emergency cricothyroidotomy.

In the operating room, a failed intubation in an anaesthetized and paralyzed patient with a contemporary failure to ventilate, using facemask ventilation or supraglottic airway device, may result in increasing hypoxemia requiring emergency cricothyroidotomy [9, 14]. In the literature several guidelines for the management of unanticipated difficult intubation are reported [15]. The onset of a CICO scenario may be the result of a poor preoperative patient assessment. CICO emergencies 
TA B L E 1. The most important screening tests for the prediction of difficult intubation.

\begin{tabular}{|c|c|}
\hline Screening tests & Explanation \\
\hline \multirow{4}{*}{$\begin{array}{l}\text { Modified Mallampati test (class III and IV are considered } \\
\text { predictive of difficult intubation) }\end{array}$} & Class I: soft palate, fauces, uvula, and pillars visible \\
\hline & Class II: soft palate, fauces, and uvula visible \\
\hline & Class III: soft palate and base of the uvula visible \\
\hline & Class IV: soft palate not visible at all \\
\hline $\begin{array}{l}\text { Thyromental distance ( } \leq 6.5 \mathrm{~cm} \text { is considered predictive } \\
\text { of difficult intubation) }\end{array}$ & $\begin{array}{l}\text { Distance from the mentum to the thyroid notch with the head fully } \\
\text { extended }\end{array}$ \\
\hline $\begin{array}{l}\text { Sternomental distance ( } \leq 13.5 \mathrm{~cm} \text { is considered predictive } \\
\text { of difficult intubation) }\end{array}$ & $\begin{array}{l}\text { Distance from the suprasternal notch to the mentum with the head } \\
\text { fully extended }\end{array}$ \\
\hline \multicolumn{2}{|l|}{$\begin{array}{l}\text { Neck circumference ( }>35 \mathrm{~cm} \text { is considered predictive of } \\
\text { difficult intubation) }\end{array}$} \\
\hline $\begin{array}{l}\text { Inter-incisor distance ( }>3.8 \mathrm{~cm} \text { is considered predictive } \\
\text { of difficult intubation) }\end{array}$ & Distance between the upper and lower incisors \\
\hline $\begin{array}{l}\text { Airway risk index (the presence of } 5 \text { or more criteria is } \\
\text { considered predictive of difficult intubation) }\end{array}$ & $\begin{array}{l}\text { It is the sum of several clinical criteria (i.e., Mallampati classifi- } \\
\text { cation, ability to prognath, mouth opening, head/neck movement, } \\
\text { body weight and previous history of difficult intubation) }\end{array}$ \\
\hline
\end{tabular}

are frequently catastrophic accounting for about $25 \%$ of all anesthesia related deaths [15]. In Table 1 are reported the most important screening tests for the prediction of a difficult intubation.

An emergency cricothyroidotomy may be also indicated in spontaneously breathing patients with an airway obstruction proximal to the subglottis caused by a wide range of diseases, such as severe maxillofacial traumas or neoplastic laryngohypopharyngeal obstructions (Table 2). In these cases, the management of CICO scenario is often performed in settings outside of the operating theatre, such as the intensive care or the emergency department and sometimes even outside the hospital setting [6].

\section{TA B LE 2. Common indications for emergency cricothyroidotomy.}

\section{Emergency cricothyroidotomy}

Severe maxillofacial trauma

Severe oropharyngeal hemorrhage

Edema of the glottis (as seen with anaphylaxis or inhalation injuries)

\section{Anatomic abnormalities}

\section{Trismus}

Unstable cervical spine fracture with contraindications for neck manipulation

Foreign body obstruction

Clenched teeth

Neoplastic laryngo-hypopharyngeal obstructions

The management of CICO scenario is a critical time and delayed or failed emergency cricothyroidotomy leads to acute cerebral hypoxia and death [16].

The CICO scenario is a rare event: in the literature its incidence varies from $1: 6000$ to $1: 13000$ routine general anesthetics $[17,18]$. In the retrospective multicenter questionnaire survey performed by Tachibana et al. [19] the incidence of CICO during a 3 -year period was $0.003 \%$ or $1: 32000$ cases conducted under general anesthesia. Rates of eFONA again vary [1] but they are approximately $1: 50000$ routine general anesthetics [20]. This means that in a hospital performing 10.000 surgeries each year a case of eFONA with the need of an emergency cricothyroidotomy would be expected once every 5 years [1]. In the intensive care units and emergency departments, however, the incidence of CICO seems to be higher (up to 10 times more frequent than in operating room) [6]. The need of an emergency surgical airway is also more frequent in trauma patients requiring emergency surgery, being reported in $0.72 \%$ of the cases [21], with severe facial trauma as the main risk factor. Furthermore, emergency cricothyroidotomy could be a rescue procedure in a pre-hospital setting too. Brown et al. [22] reported a series of 4,871 emergency airway encounters of which $91.7 \%$ were finally managed with intubation. In the other cases, 168 received an extraglottic device and 35 a cricothyroidotomy $(0.7 \% 33$ out of 35 were successful). Nevertheless, the CICO scenario remains very rare and many anesthesiologists, intensivists and surgeons may not have performed an emergency cricothyroidotomy during their training [23-25]. For this reason, educational programs with training on high fidelity manikins or advanced simulators or cadaveric models should be implemented in every hospital [23].

There are a few of absolute contraindications for emergency cricothyroidotomy: the presence of a post-traumatic transection of the airway with retraction of the trachea in the mediastinum, a significant cricoid or laryngeal fracture and a neoplastic or inflammatory stenosis of the subglottic larynx. Relative contraindications for emergency cricothyroidotomy are all that situations characterized by the presence of anatomic "barriers" such as large goiters or a laryngeal tumor with invasion of the cricothyroid membrane. The useful mnemonic [26] 


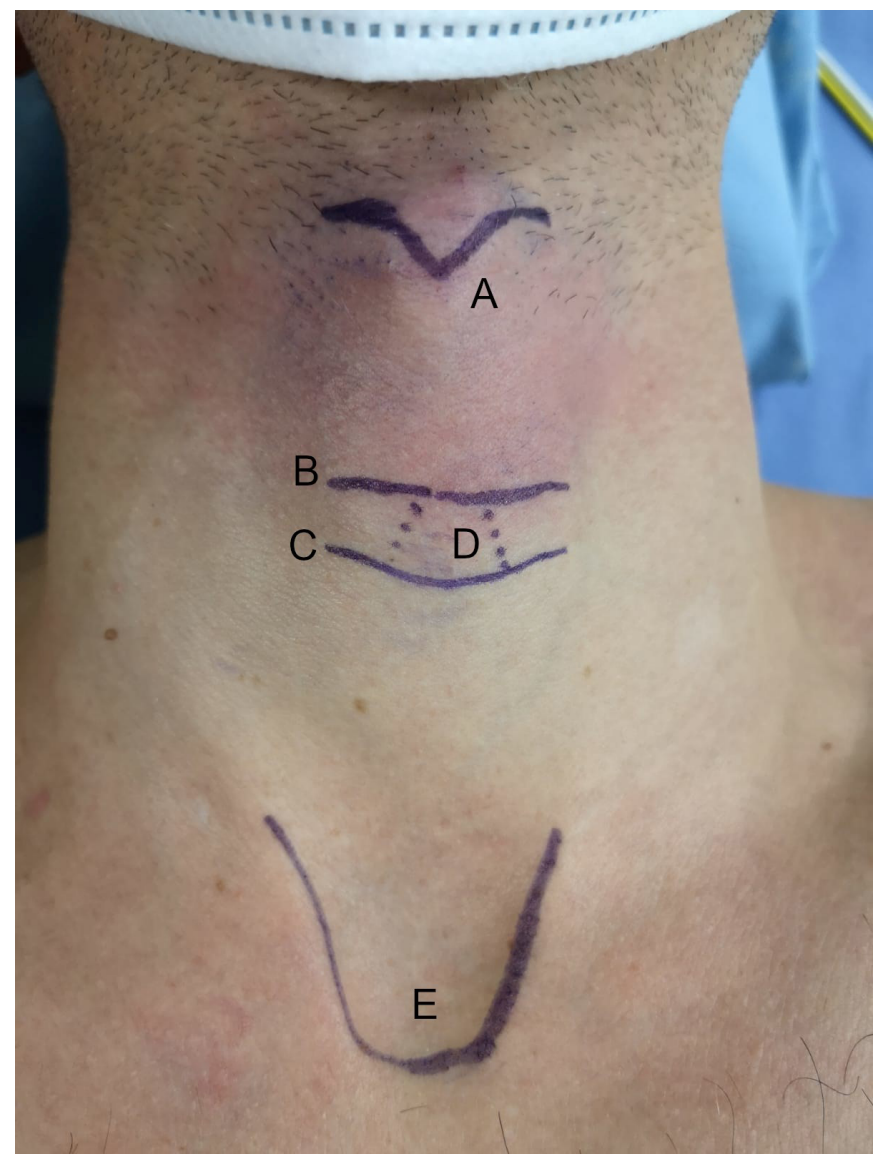

F I G U R E 1. Neck, anterior view. (A), superior border of thyroid cartilage; (B), inferior border of thyroid cartilage; (C), superior border of cricoid cartilage; (D), cricothyroid membrane; (E), suprasternal notch.

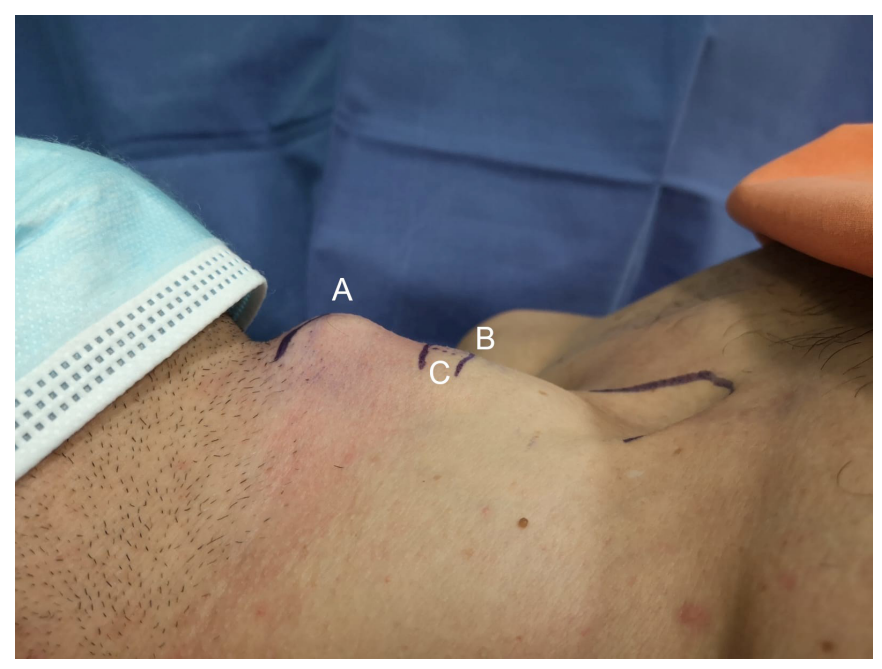

F I G U RE 2. Neck, lateral view. (A), thyroid prominence; (B), cricoid prominence; (C), cricothyroid membrane.

for difficult cricothyroidotomy SHORT (surgery, hematoma, obesity, radiation, tumor/trauma) should be taken in account.

\section{Surface and surgical anatomy}

A thorough knowledge of laryngo-tracheal anatomy as well as its anatomical variants is essential for a successful performance of cricothyroidotomy. According to Boon et al. [23, 27] an intimate familiarity of the laryngeal anatomy is essential, not only to lessen tube misplacement and other complications of the procedure, but also to reduce anxiety among providers.

The first anatomical landmark in the anterior midline of the neck superiorly is the "Adam's apple" or thyroid prominence. Moving inferiorly the next prominence (second landmark) is the cricoid cartilage. Above the cricoid and between the thyroid and cricoid prominence, the finger can "identify" the depression of the cricothyroid membrane (third landmark; Figs. 1,2).

A cricothyroidotomy enters the subglottic larynx in the midline through the median cricothyroid ligament of the cricothyroid membrane. The cricothyroid membrane is a trapezoidal fibro-elastic structure bordered laterally by the cricothyroid muscles. The medial part of cricothyroid membrane is known as the median cricothyroid ligament. The incision passes through skin, subcutaneous, median cricothyroid ligament of cricothyroid membrane and mucosa of the subglottic larynx. Then the endotracheal tube or tracheostomy cannula passes through the cricoid ring and the trachea. The only vessels that may be encountered are the cricothyroid arteries that course across the upper part of the cricothyroid membrane. For this reason, the cricothyroidotomy should always be done cutting the cricothyroid membrane along the superior margin of the cricoid cartilage. The section of the cricothyroid artery may explain why some cricothyroidotomy are bloody whereas others are not [6].

The dimensions of the cricothyroid membrane vary in adults between 9-10 $\mathrm{mm}$ high [23] and 22-33 $\mathrm{mm}$ wide (but the space between the cricothyroid muscles is much less, being about $8.2 \mathrm{~mm}$ ) [27]. Therefore, to avoid injuries to the subglottic larynx, a tube of no more than $8 \mathrm{~mm}$ outer diameter should be used. The "Difficult Airway Society" in fact suggests a 6.0 $\mathrm{mm}$ inner diameter tracheal tube or a cannula having an outer diameter of up to $8 \mathrm{~mm}[15,28]$. In the largest available series of CT scan measurements, Nutbeam et al. [28] found that the height of the cricothyroid membrane was smaller than that previously reported. In their study a total of 482 trauma scans were assessed. The mean height of the cricothyroid membrane as measured by two radiologists was $7.89 \mathrm{~mm}$ and $7.88 \mathrm{~mm}$ in male patients and $6.00 \mathrm{~mm}$ and $5.92 \mathrm{~mm}$ in female patients. The height of the cricothyroid membrane moreover increases (by up to 20\%) when the head and the neck are placed in fully extension [23, 29, 30], confirming the necessity of performing cricothyroidotomy in this position [30]. It is likely that a decrease in cricothyroid membrane static height as well as in its dynamic height (achieved by extension) may be observed due to calcification associated with the ageing process [28].

Several studies have shown that in an emergency situation the identification of the cricothyroid membrane by palpation may be difficult or impossible, particularly in females and in obese patients [31-33]. Careful attention should be paid in pregnant women with respiratory disorders, considering the difficulty to the laryngeal digital examination due to pregnancy-related anatomy modifications. The traditional method of palpating the cricothyroid membrane is based on palpation of the thyroid cartilage and the depression between the thyroid and cricoid cartilage. This can be easily performed 


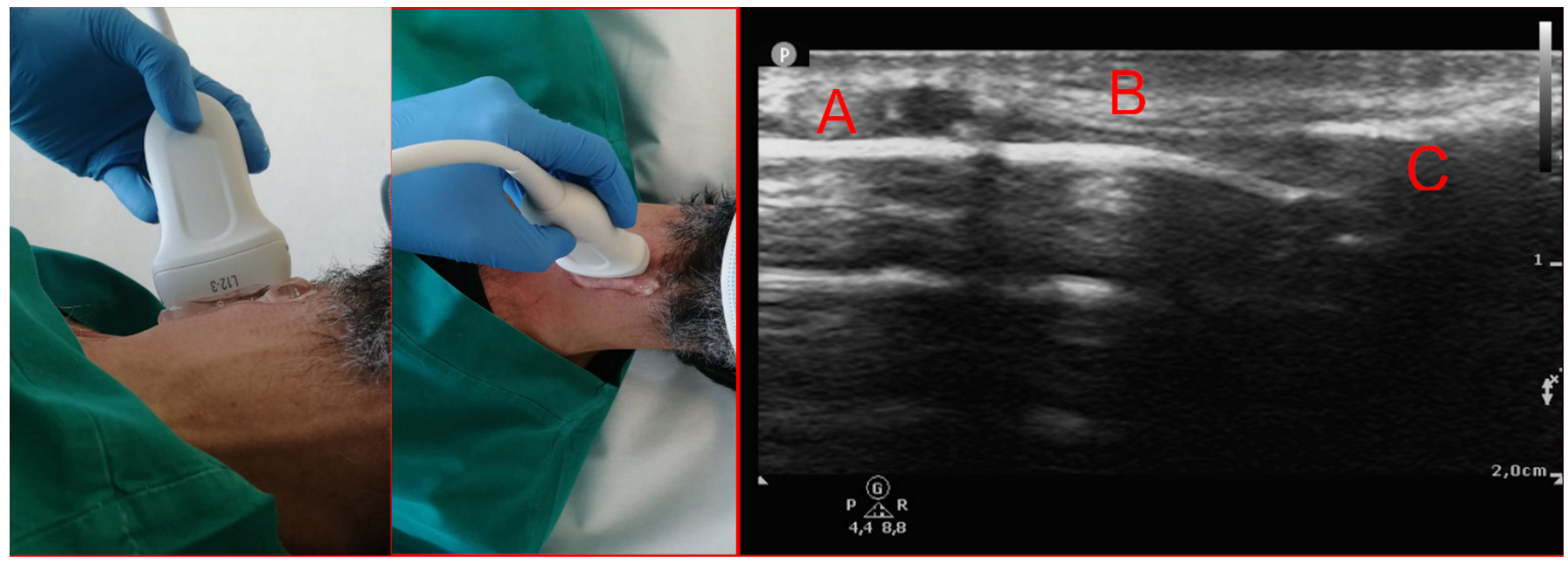

F I G U R E 3. Ultrasound localization of the cricothyroid membrane. Left and central images: lateral and anterior view of the ultrasound neck examination. Right image: ultrasound view; (A) cricoid cartilage, (B) cricothyroid membrane, (C) thyroid cartilage.

in males but not in obese subjects with a fat neck. In females moreover there is no Adam's apple and the thyroid and cricoid cartilages have equal prominence [34]. In the study by Eliott et al. [35] the capacity of 18 anesthesiologists to identify the cricothyroid membrane in 6 subjects was evaluated. The authors reported that this group of anesthesiologists was able to locate the membrane only in the $30 \%$ of the cases. Other prospective studies have demonstrated that clinicians (including surgeons) can successfully palpate the membrane in only $70 \%$ of patients, number that falls to $30 \%$ if the patient is obese [36-39].

To avoid the misidentification of the cricothyroid membrane with the misplacement of the cannula, Levitan [34] described a method for identifying the laryngeal structures called "laryngeal handshake". As reported by this author, the maneuver is performed with the thumb and the middle finger of the dominant hand identifying the hyoid and thyroid laminae, stabilizing the larynx between these two fingers, and moving down the neck to palpate the cricothyroid membrane with the index finger. After that the index finger of the dominant hand is substituted by that of the non-dominant hand and the dominant hand stabilized on the sternum is used to make the procedure $[16,34]$.

The "Difficult Airway Society" [15] suggests the use of the "laryngeal handshake" as a method for identifying the cricothyroid membrane. Up today, however, the efficacy of this technique has been assessed in the study by Drewn and McCaul [16] only. In this study, five clinicians were asked to identify the cricothyroid membrane using the "laryngeal handshake" method in a total of 45 anaesthetized females (Group L) and by conventional palpation in 45 controls (Group P). Successful identification of the cricothyroid membrane occurred in $62 \%$ and $33 \%$ of Group L and Group P patients, respectively. The midline was accurately identified more frequently in Group L than in Group P patients. The mean time of the cricothyroid membrane identification was greater in Group L at $31 \mathrm{~s}$ compared with Group P in which took 18 s. The authors concluded that the "laryngeal handshake" tech- nique is more accurate but takes longer than the conventional palpation technique in locating the cricothyroid membrane and the midline.

The use of ultrasound to precisely identify the location of the cricothyroid membrane (Fig. 3) has been reported in the literature in several studies [40-42]. Although some authors recommend the routine ultrasonographic identification of the membrane in patients without anatomical landmarks [40], the use of this technique is not recommended in emergency situations [1]. However, in selected patients with an anticipated difficult airway and a cricothyroid membrane difficult to be palpate, a good rule may be that to use ultrasound prior to the induction of anesthesia to identify the site of the membrane that will be marked with a pen for a later use, if should the need arise [1].

\section{Techniques}

Three different techniques of cricothyroidotomy are in use today: needle, percutaneous and surgical. Some debate exists about which procedure is superior over all other techniques in terms of successful cannula placement and complication rates [7, 43-45]. However, it is likely that each procedure should be used in a specific clinical scenario to make the best use of its technical characteristics.

Needle cricothyroidotomy is the rescue technique of choice for the failed airway in pediatric population [46]. It can be employed also in extreme situations when an emergency cricothyroidotomy set or the equipment to perform a surgical cricothyroidotomy are not available. In the adults, percutaneous cricothyroidotomy is mostly used in the operating room for the management of a CICO emergency while surgical cricothyroidotomy is more often performed in settings outside of the operating theatre (intensive care and the emergency departments) or in pre-hospital settings.

In all these three techniques the first step is to stabilize the thyroid cartilage with the first and third fingers of the nondominant hand leaving the second finger free to identify the cricothyroid membrane [47]. The second step should be, if 
there is enough time, to inject $2 \%$ lidocaine into the skin, subcutaneous tissue and through the cricothyroid membrane into the subglottic larynx to anaesthetize the airway. Although a cricothyroidotomy may be performed also with the neck in a neutral position, the better exposure of the surgical field is obtained with the patient's neck extended or hyper-extended. As the cricothyroidotomy may be a real emergency procedure, often there is no time for the preparation of a standard sterile field.

\subsection{Needle cricothyroidotomy}

A large bore (12-14 gauge) intravenous cannula or the cannula of a specifically designed needle cricothyroidotomy set is introduced at a $45^{\circ}$ caudal angle into the trachea through the superior margin of the cricoid ring percutaneously, with negative pressure aspiration through a fluid-filled syringe. Once the needle traverses the cricothyroid membrane air bubbles appear in the syringe. The cannula is then advanced into the airway while the needle must be withdrawn. To deliver both high flow and high pressure to the patient to overcome the cannula resistance [1], a jet insufflator should be attached to the cannula. Jet ventilation is not suitable for long-term ventilation [4] as it does not permit adequate ventilation and hence leads to hypercapnia [47]. In adult patients the standard practice is to intubate or convert in a formal tracheostomy as soon as possible. Although reports of successful ventilation with standard ventilators exist [1, 48, 49], jet ventilation seems to be the only temporary technique for emergency ventilation through a needle cricothyroidotomy usable in practice. However, needle cricothyroidotomy can be quickly converted to surgical cricothyroidotomy $[6,50]$.

\subsection{Percutaneous cricothyroidotomy}

Percutaneous cricothyroidotomy can be performed using a Seldinger or cannula-over-trocar technique. These two techniques were recently compared by Metterlein et al. [51] in an animal model in terms of insertion time, success rate and complications. With the Seldinger technique successful placement was possible in all attempts. The cannula-overtrocar technique was successful in $63 \%$ and had a higher complication rate $(75 \%$ vs $13 \%)$. The cannula-over-trocar technique allowed a faster procedure ( $32 \mathrm{~s}$ vs $53 \mathrm{~s}$ ). The authors concluded that the Seldinger method seem to be the more reliable technique leading to fewer complications. However, the cannula-over-trocar technique was faster to perform. The Seldinger cricothyroidotomy is, in fact, considered the most acceptable technique to anesthesiologists [1, 52]. It can be performed using a specifically designed percutaneous cricothyroidotomy set. A small stab incision in the skin over the cricothyroid membrane is performed. A needle attached to a syringe filled with normal saline is passed through the cricothyroid membrane and then directed caudally at $45^{\circ}$. As the needle is into the subglottic larynx air bubbles will appear in the syringe. The syringe is disconnected from the needle and a guide wire is inserted through the needle into the airway. Once the guide wire is into the trachea the needle is removed. The dilator and the tracheostomy cannula are jointly advanced over the guide wire into the airway. The dilator and the guide- wire are removed leaving the tracheostomy cannula in situ. The tracheostomy cannula is then secured with stitches or a tracheostomy tape.

\subsection{Surgical cricothyroidotomy}

As advocated by both the Advanced Trauma Life Support and the Difficult Airway Society [44, 53], a 1-2 cm horizontal stab incision is made using a no. 10/11 blade scalpel on the skin over the cricothyroid membrane. After having clearly felt the cricothyroid membrane with the non-dominant index finger, a $1 \mathrm{~cm}$ transverse incision is made through the membrane along the superior border of the cricoid ring. The tract is dilated passing a curved haemostat through the incision and angling it caudally at $45^{\circ}$ to avoid the lesion of the posterior wall of the trachea. Alternatively, a no. 10 blade scalpel is inserted into the airway along the superior edge of the cricoid and then rotated $90^{\circ}$, as suggested by the Difficult Airway Society [44]. A tracheostomy cannula is then inserted into the airway either directly or over a bougie. After having confirmed the correct placement of the tracheostomy cannula, the ventilation is initiated. The tracheostomy cannula is then secured with stitches or a tracheostomy tape.

If the anatomical landmarks are not easily identified (fat necks), it may be better to perform a $3 \mathrm{~cm}$ vertical midline skin incision in order to try to identify, with the non-dominant index finger moved from up to down and from side to side, the cricothyroid membrane and the superior edge of the cricoid. If this is not feasible the incision of the skin and the subcutaneous tissue may be extended. The Difficult Airway Society published a consensus-based recommendation that an $8-10 \mathrm{~cm}$ vertical incision should be performed to access the cricothyroid membrane when the surface anatomy is not well defined [15], but the incision commencement point as well as the incision length are not reported. Fennessy et al. [54] measured, using ultrasound in 80 healthy subjects, the distance between the suprasternal notch and the cricothyroid membrane to determine a theoretical optimal incision position and length. The authors showed that an $8 \mathrm{~cm}$ incision commencing $3 \mathrm{~cm}$ above the suprasternal notch would include all cricothyroid membrane locations in the extended neck position.

The studies reporting the speed of execution of cricothyroidotomy are not comparable because they are not standardized in terms of provider level, airway experience and type of procedure [6, 7, 55-67]. In these studies, however, surgical cricothyroidotomy seems to be faster than percutaneous cricothyroidotomy when performed for gaining access to the airway in emergency settings. On the other hand, surgical technique could be more frequently related to inadvertent contamination from devices and/or aerosol contamination, as reported in a study by Chua et al. [68]. The choice of the eFONA method should take into account these implications when managing infective respiratory diseases, especially during the coronavirus 2019 pandemic. The time difference between the performances of the two techniques is in seconds rather than in minutes [69] and it is likely that this small time factor doesn't have any impact on mortality [1]. In a randomized trial involving sheep models, Heard et al. [70] demonstrated a better performance in oxygen delivery using 
the scalpel-finger-cannula technique as compared to scalpelfinger-bougie, when managing a simulated CICO scenario. In another animal study with porcine models, Umek et al. [71] compared the success rate of rescue oxygenation (defined as arterial oxygen saturation $>90 \% 5 \mathrm{~min}$ after the beginning of oxygenation) between surgical scalpel-bougie technique and percutaneous cannula technique. A significantly higher success rate was observed for the surgical technique (83\% vs $18 \%$ ), but it was related to a more frequent iatrogenic trauma (75\% vs 27\%). Furthermore, Heymans et al. [72] tested the performance of 20 medical personnel, naive to surgical airway technique, in establishing an emergency cricothyrotomy in cadavers, using three different techniques (Seldinger, trocar-like insertion needle, surgical). Participants established emergency cricothyrotomy more efficiently and safely with the surgical procedure than with the other two commonly used techniques.

Today, there is no clear evidence demonstrating superiority of a particular cricothyroidotomy technique [69]. The choice of cricothyroidotomy techniques depends on several factors: provider preference (based on training), anatomical knowledge, presence or loss of the anatomical landmarks, age of patients, commercial cricothyroidotomy kit availability, level of familiarity with a given technique, and setting (hospital or pre-hospital). All the airway practitioners (in particular anesthesiologists and surgeons) should be aware about all types of cricothyroidotomy and able to perform at least one. A multidisciplinary hospital team should be involved in every eFONA procedure, in order to use the technique with the best chances of success in every clinical scenario.

\section{Success rate of cricothyroidotomy}

The success rate of cricothyroidotomy varies widely among the studies reported in the literature according to clinical settings. In the study by Kwon et al. [24] the success rate of cricothyroidotomy performed in an emergency department and the survival rate in the CICO scenario were $74 \%$ and $48 \%$, respectively. Also, in the study by Paix and Griggs [73] the success rate of cricothyroidotomy was not very high. These authors, two medical specialists with backgrounds in anesthesia and aeromedical retrieval, performed 24 surgical cricothyroidotomy mainly under adverse pre-hospital conditions over a combined 40 years of practice and reported a success rate of $63 \%$. In the meta-analysis by Hubble et al. [74], however, the success rate in a pre-hospital series of 485 patients including 18 studies was higher (90.5\%).

The study by Langvad et al. [7] underscores a very important point, namely that focusing on cricothyroidotomy success rate is not enough because also time aspects are essential. Most patients who require emergency cricothyroidotomy in the operating rooms, emergency departments, intensive care units or pre-hospital settings have failed several endotracheal intubation attempts and are non-ventilable and critically hypoxic. The definition of cricothyroidotomy success should include the time used to secure the airway. In a CICO scenario when endotracheal intubation and other airway devices have failed, and the patient has already started to deteriorate, the useful time to establish a secure airway performing an emergency cricothyroidotomy with chances of success ranges between 40 and 60 seconds $[8,64,75]$, beyond which the risk of severe hypoxia becomes too high. Another outcome indicator is the rise in blood oxygen levels: an arterial oxygen saturation $>$ $90 \%$ five minutes after the beginning of oxygenation should be the minimal target. Despite a fast airway control, however, many patients will die for the severity of their diseases. For this reason, a positive patient outcome is not a requirement for cricothyroidotomy success [6].

The main reason for cricothyroidotomy failure is the lack of anatomical landmarks, with the misplacement of the cannula and/or the performance of the procedure beyond 60 seconds $[7,76]$. However, the cricothyroidotomy failure is often due to the stress of the operator in a life-threatening scenario [76]. Despite the availability of several effective methods of cricothyroidotomy training which provide an adequate skill preparation, none of them prepares providers to the highstress application of the skill in life threatening situations [6]. A recent study on difficult tracheal intubation events using the Anesthesia Closed Claims Project database [77] highlighted poor outcomes in malpractice claims related to difficult tracheal intubation, mainly due to inadequate airway planning and judgment errors. Authors emphasized the need to improve both practitioner skills and systems response in CICO scenarios, to improve patients' outcomes.

\section{Complications}

The reported complications vary from $0 \%$ to as high as $54 \%$ [4] depending on the clinical scenario, provider level of training and presence or lack of anatomical landmarks.

Complications are more frequent in pre-hospital than in inhospital procedures owing to inadequate lighting, inability to have a sterile field and lack of equipment [6]. Complications were noted in $14 \%$ and $54 \%$ of all in-hospital and pre-hospital cricothyroidotomies, respectively [78].

Cricothyroid complications can be classified in immediate and delayed. The most important immediate and delayed complications and the strategies to remedy or mitigate them are reported in Table 3.

\section{Cricothyroidotomy: the thoracic surgeon's perspective}

The emergent access to the airway obtained through the cricothyroid membrane by cricothyroidotomy is the final rescue maneuver in scenarios of difficult airway management [43, 79]. Cricothyroidotomy is preferable for the majority of patients requiring a prompt access to a compromised and inaccessible airway because it is easier and quicker to perform than an emergency tracheostomy [74, 80, 81]. Even for experienced airway surgeons (head and neck and thoracic surgeons) with large experience in tracheostomy performance, cricothyroidotomy is the technique of choice in $\mathrm{CICO}$ emergencies for the easy anatomical landmarks and the proximity of the cricothyroid membrane to the neck skin [82].

The emergency care of patients with difficult airway requires effective participation of an interprofessional teamwork. Optimal care depends not only on the medical or surgical aspects but also on the delivery of care within the context of 
TA B L E 3. Cricothyroidotomy complications and strategies for their treatment.

\begin{tabular}{|c|c|c|}
\hline Immediate complication & Cause & Strategy \\
\hline \multirow[t]{3}{*}{ Creation of a paratracheal false tract } & Misplaced skin incision & Identify again the anatomical landmarks \\
\hline & & Extend a vertical incision superiorly and/or inferiorly \\
\hline & & If time, use ultrasound to help landmarks identification \\
\hline \multicolumn{2}{|c|}{$\begin{array}{l}\text { Incorrect cannula placement through the Misplaced skin incision } \\
\text { thyrohyoid membrane }\end{array}$} & $\begin{array}{l}\text { Commence ventilation. If it is possible to ventilate the lungs (check with } \\
\text { a pediatric fiberscope that the cannula is into the trachea) convert the } \\
\text { procedure in a formal tracheostomy (in-hospital setting). If the cannula } \\
\text { doesn't work, remove it and repeat the procedure (in- and pre-hospital } \\
\text { setting) }\end{array}$ \\
\hline
\end{tabular}

Posterior tracheal wall perforation into Insertion of the cannula into the airway with an angle of more Remove the cannula and repeat the procedure

esophagus

than $45^{\circ}$ (percutaneous cricothyroidotomy) or a perpendicular

scalpel position when stabbing the cricothyroid membrane or

excessive depth of scalpel penetration through the cricothyroid

membrane (surgical cricothyroidotomy)

Bleeding (minor)
Cricothyroid arteries injury (incision of the superior half of the

cricothyroid membrane)

Bleeding (severe)

Lacerations of a very large cricothyroid artery and of the anterior Severe bleeding is very rare. However, if occur packing at the site should jugular veins provide control. If not, look for other bleeding causes

Cannula misplacement resulting in endo- Insertion of the endotracheal tube too deep or angling the tube Retract the tube until chest movement and breath sounds are appreciated bronchial or endopharyngeal intubation inferiorly

Fracture of the cricoid and/or thyroid It may occur due to an oversized tube being forced through the If the placement of an oversized tube is impossible, insert a bougie cartilage cricothyroid membrane through the tract into the airway. Subsequently, insert a smaller cannula by railroading it over the bougie

Glottic or subglottic stenosis

Fibrosis, granulomas and perichondritis of subglottic larynx Convert an emergency cricothyroidotomy in a formal tracheostomy as (reported only in long-term cricothyroidotomies) soon as it is possible

Dysphonia

Misplaced skin incision and damage to vocal cords

Remove the cannula and repeat the procedure

Persistent stoma and tracheoesophageal Reported only in long-term cricothyroidotomies

Convert an emergency cricothyroidotomy in a formal tracheostomy as fistula soon as it is possible 
Can't intubate, can’t oxygenate (CICO) scenario: Cuneo Hospital's emergency protocol

$\mathrm{CICO}$

- Declare CICO

- Call for help

- Extend patient neck

- Stand on right side (left side for left handers)

\title{
EMERGENCY FRONT OF NECK ACCESS (SURGICAL CRICOTHYROIDOTOMY)
}

\author{
Equipment: Scalpel (number 10 blade), Bougie, 6.0 cuffed tube. \\ Technique: \\ 1. Laryngeal hand scale to localize the cricothyroid membrane \\ 2. Stabilise the trachea with the non-dominant hand \\ 3. Incision: \\ a. Palpable cricothyroid membrane: $3-4 \mathrm{~cm}$ transverse skin incision along the superior \\ border of the cricothyroid membrane \\ b. Impalpable cricoth yroid membrane: $8-10 \mathrm{~cm}$ vertical midline skin incision starting \\ about $3 \mathrm{~cm}$ above the sternal nock \\ 4. Insert the lubricated tube into the airway over a bougie \\ 5. Ventilate, inflate cuff and confirm position with capnography \\ 6. Secure tube
}

F I G U R E 4. Can't intubate, can't oxygenate (CICO) scenario: Cuneo Hospital's emergency protocol.

a difficult airway team including anesthesiologists, other medical specialties and nurses. The concept of a multidisciplinary airway rapid response team was developed as an approach to assemble specialized equipment and diverse clinical expertise for securing of the airway when difficulty is encountered with standard maneuvers in the shortest time possible [83-85]. The multidisciplinary airway rapid response team may mitigate the risk of death and other complications related to the failed airway intubation scenario [83].

From a technical point of view cricothyroidotomy is a simple procedure that can be performed not only by physicians but also by other trained healthcare providers [5]. As a part of an interprofessional teamwork (emergency difficult airway team), the thoracic surgeon may have a unique role in regard to his more experienced background in laryngo-tracheal anatomy [86]. For this reason, he may often be called upon to manage a CICO emergency in many settings, such as emergency wards, intensive care units and operating rooms. It is therefore evident the importance to have in each hospital a standardized protocol for the emergent access to the airway in order to reduce time to ventilation and improve patient successful chances.

In 2018 anesthesiologists and thoracic surgeons of our hospital (a tertiary care hospital with $24 / 7$ in-house specialist coverage) adopted a unified multidisciplinary approach to the in-hospital CICO scenarios. Our eFONA protocol for difficult airway management is reported in Fig. 4. When a CICO scenario is encountered, the anesthesiologist declares the decision to perform a cricothyroidotomy and try to continue to oxygenate via face mask, nasal cannula and SAD. If there is enough time the thoracic surgeon is called in the operating room or anywhere in the hospital. Otherwise, the cricothyroidotomy is performed by the anesthesiologist.

We have adopted the surgical cricothyroidotomy as the technique of choice in adults to provide oxygenation to patients in CICO scenario, not only because it can be performed with a simple surgical set, but also because of its higher success rates compared to the percutaneous technique [15, 81, 82]. Both when a horizontal or a vertical incision is used, the airway is entered making a transverse incision through the cricothyroid membrane along the superior edge of the cricoid cartilage in order to reduce the risk of bleeding. The small endotracheal tube is inserted into the airway over a bougie. The emergency surgical cricothyroidotomy equipment is available in all the operating rooms of our hospital. Furthermore, in order to have a readily available airway-skilled interprofessional staffs, regular multidisciplinary trainings with manikins are organized to improve and maintain the performance of emergency cricothyroidotomy.

\section{AUTHOR CONTRIBUTIONS}

FM: literature searching, article drafting, final revision and approval. MV: article drafting, final revision and approval. DT: article drafting, final revision and approval. AG: article drafting, final revision and approval. CD: article drafting, final revision and approval. AL: study coordination, article drafting, final revision and approval. GM: study design and coordination, literature searching, article drafting, final revision and approval. 


\section{FUNDING}

This research received no external funding.

\section{CONFLICT OF INTEREST}

The authors declare no conflict of interest.

\section{REFERENCES}

[1] Melachuri V, Ince M. Emergency front of neck access. Indian Journal of Respiratory Care. 2017; 6: 793.

[2] Schroeder AA. Cricothyroidotomy: when, why, and why not? American Journal of Otolaryngology. 2000; 21: 195-201.

[3] Brantigan CO, Grow JB. Cricothyroidotomy: elective use in respiratory problems requiring tracheotomy. The Journal of Thoracic and Cardiovascular Surgery. 1976; 71: 72-81.

[4] McKenna P, Desai NM, Morley EJ. Cricothyrotomy. StatPearls Publishing. 2020.

[5] Šimunjak T, Goranović T, Šimunjak B. Cricothyrotomy - urgent access to the airway, when and how? Acta Medica Croatica. 2018; 72: 57-62.

[6] Hessert MJ, Bennett BL. Optimizing emergent surgical cricothyrotomy for use in austere environments. Wilderness \& Environmental Medicine. 2013; 24: 53-66.

[7] Langvad S, Hyldmo P, Nakstad A, Vist G, Sandberg M. Emergency cricothyrotomy - a systematic review. Scandinavian Journal of Trauma, Resuscitation and Emergency Medicine. 2013; 21: 43.

[8] DiGiacomo C, Neshat KK, Angus LDG, Penna K, Sadoff RS, Shaftan GW. Emergency cricothyrotomy. Military Medicine. 2003; 168: 541544.

[9] Rao S, Mendoca C. Airway emergency - management of can't intubate can't oxygenate scenario. Journal of Anaesthesia Practice. 2006.

[10] Civitarese F. Cricothyrotomy (CT) as a primary airway maneuver vs. an airway salvage technique. emDOCs. 2015.

[11] NAP4, Executive summary - The Royal College of Anaesthetists. Major complications of airway management in the United Kingdom. 2011. Available at: https://www.rcoa.ac.uk/media/6741 (Accessed: 07 March 2021).

[12] Cook TM, Woodall N, Harper J, Benger J. Major complications of airway management in the UK: results of the Fourth National Audit Project of the Royal College of Anaesthetists and the Difficult Airway Society. Part 2: intensive care and emergency departments. British Journal of Anaesthesia. 2011; 106: 632-642.

[13] Cook TM, Woodall N, Frerk C. A national survey of the impact of NAP4 on airway management practice in United Kingdom hospitals: closing the safety gap in anaesthesia, intensive care and the emergency department. British Journal of Anaesthesia. 2016; 117: 182-190.

[14] Govender V. Cricothyroidotomy and the difficult airway. Anaesthetics. 2016.

${ }^{[15]}$ Frerk C, Mitchell VS, McNarry AF, Mendonca C, Bhagrath R, Patel A, et al. Difficult Airway Society 2015 guidelines for management of unanticipated difficult intubation in adults. British Journal of Anaesthesia. 2016; 115: 827-848.

[16] Drew T, McCaul CL. Laryngeal handshake technique in locating the cricothyroid membrane: a non-randomised comparative study. British Journal of Anaesthesia. 2018; 121: 1173-1178.

[17] Nagaro T, Yorozuya T, Sotani M, Adachi N, Tabo E, Arai T, et al. Survey of patients whose lungs could not be ventilated and whose trachea could not be intubated in university hospitals in Japan. Journal of Anesthesia. 2003; 17: 232-240.

[18] Kheterpal S, Martin L, Shanks AM, Tremper KK. Prediction and outcames of impossible mask ventilation: a review of 50.000 anesthetics. Anestesiology. 2009; 110: 891-897.

[19] Tachibana N, Niiyama Y, Yamakage M. Incidence of cannot intubatecannot ventilate (CICV): results of a 3-year retrospective multicenter clinical study in a network of university hospitals. Journal of Anesthesia. 2015; 29: 326-330.

[20] Timmermann A, Chrimes N, Hagberg CA. Need to consider human factors when determining first-line technique for emergency front-ofneck access. British Journal of Anaesthesia. 2016; 117: 5-7.

[21] Ono Y, Yokoyama H, Matsumoto A, Kumada Y, Shinohara K, Tase C. Surgical airways for trauma patients in an emergency surgical setting: 11 years' experience at a teaching hospital in Japan. Journal of Anesthesia. 2013; 27: 832-837.

[22] Brown III C, Cox K, Hurwitz S, Walls R. 4,871 Emergency airway encounters by air medical providers: a report of the air transport emergency airway management (NEAR VI: "A-TEAM") project. Western Journal of Emergency Medicine. 2014; 15: 188-193.

[23] Boon JM, Abrahams PH, Meiring JH, Welch T. Cricothyroidotomy: a clinical anatomy review. Clinical Anatomy. 2004; 17: 478-486.

[24] Kwon YS, Lee CA, Park S, Ha SO, Sim YS, Baek MS. Incidence and outcomes of cricothyrotomy in the "cannot intubate, cannot oxygenate" situation. Medicine. 2019; 98: e17713.

[25] Asai T. Surgical cricothyrotomy, rather than percutaneous cricothyrotomy, in "cannot intubate, cannot oxygenate" situation. Anesthesiology. 2016; 125: 269-271.

[26] Walls R, Murphy M. Chapter 7: identification of the difficult airway. Manual of Emergency Airway Management. 3rd edn. Lippincott Williams \& Wilkins. 2008.

[27] Dover K, Howdieshell TR, Colborn GL. The dimensions and vascular anatomy of the cricothyroid membrane: Relevance to emergent surgical airway access. Clinical Anatomy. 1996; 9: 291-295.

[28] Nutbeam T, Clarke R, Luff T, Enki D, Gay D. The height of the cricothyroid membrane on computed tomography scans in trauma patients. Anaesthesia. 2017; 72: 987-992.

[29] Bennett JD, Guha SC, Sankar AB. Cricothyrotomy: the anatomical basis. Journal of the Royal College of Surgeons of Edinburgh. 1996; 41: 57-60.

[30] Dixit A, Ramaswamy KK, Perera S, Sukumar V, Frerk C. Impact of change in head and neck position on ultrasound localisation of the cricothyroid membrane: an observational study. Anaesthesia. 2019; 74: $29-32$.

[31] Lamb A, Zhang J, Hung O, Flemming B, Mullen T, Bissell MB, et al. Accuracy of identifying the cricothyroid membrane by anesthesia trainees and staff in a Canadian institution. Canadian Journal of Anesthesia. 2015; 62: 495-503.

[32] Kristensen MS, Teoh WH, Rudolph SS, Tvede MF, Hesselfeldt R, Børglum J, et al. Structured approach to ultrasound-guided identification of the cricothyroid membrane: a randomized comparison with the palpation method in the morbidly obese. British Journal of Anaesthesia. 2015; 114: 1003-1004.

[33] Aslani A, Ng S, Hurley M, McCarthy KF, McNicholas M, McCaul CL. Accuracy of identification of the cricothyroid membrane in female subjects using palpation. Anesthesia \& Analgesia. 2012; 114: 987-992.

[34] Levitan RM. Tips and tricks for performing cricothyrotomy. ACEP Now. 2014.

[35] Elliott DSJ, Baker PA, Scott MR, Birch CW, Thompson JMD. Accuracy of surface landmark identification for cannula cricothyroidotomy. Anaesthesia. 2010; 65: 889-894.

[36] Hiller KN, Karni RJ, Cai C, Holcomb JB, Hagberg CA. Comparing success rates of anesthesia providers versus trauma surgeons in their use of palpation to identify the cricothyroid membrane in female subjects: a prospective observational study. Canadian Journal of Anesthesia. 2016; 63: 807-817.

[37] You-Ten KE, Desai D, Postonogova T, Siddiqui N. Accuracy of conventional digital palpation and ultrasound of the cricothyroid membrane in obese women in labour. Anaesthesia. 2015; 70: 1230-1234.

[38] Y1ldız G, Göksu E, Şenfer A, Kaplan A. Comparison of ultrasonography and surface landmarks in detecting the localization for cricothyroidotomy. The American Journal of Emergency Medicine. 2016; 34: 254-256.

[39] Hsiao J, Pacheco-Fowler V. Videos in clinical medicine. Cricothyroidotomy. The New England Journal of Medicine. 2008; 358: e25.

[40] Kristensen MS, Teoh WH, Rudolph SS. Ultrasonographic identification of the cricothyroid membrane: best evidence, techniques, and clinical impact. British Journal of Anaesthesia. 2016; 117: i39-i48.

[41] Mallin M, Curtis K, Dawson M, Ockerse P, Ahern M. Accuracy of ultrasound-guided marking of the cricothyroid membrane before simulated failed intubation. The American Journal of Emergency Medicine. 2014; 32: 61-63. 
[42] Siddiqui N, Arzola C, Friedman Z, Guerina L, You-Ten KE. Ultrasound improves cricothyrotomy success in cadavers with poorly defined neck anatomy: a randomized control trial. Anesthesiology. 2015; 123: 1033 1041.

[43] Henderson JJ, Popat MT, Latto IP, Pearce AC. Difficult Airway Society guidelines for management of the unanticipated difficult intubation. Anaesthesia. 2004; 59: 675-694.

[44] Frerk C, Mitchell VS, McNarry AF, Mendonca C, Bhagrath R, Patel A, et al. Difficult Airway Society 2015 guidelines for management of unanticipated difficult intubation in adults. British Journal of Anaesthesia. 2015; 115: 827-848.

[45] Scrase I, Woollard M. Needle vs surgical cricothyroidotomy: a short cut to effective ventilation. Anaesthesia. 2006; 61: 962-974.

[46] Coté CJ, Hartnick CJ. Pediatric transtracheal and cricothyrotomy airway devices for emergency use: which are appropriate for infants and children? Pediatric Anesthesia. 2009; 19: 66-76.

[47] Fagan J. Cricothyroidotomy \& needle cricothyrotomy. The open access atlas of otolaryngology, Head \& Neck Operative Surgery. Johan Fagan. 2016. Available at: https : //doi .org/10.15641/0-7992-2534-1

[48] Bould MD, Bearfield P. Techniques for emergency ventilation through a needle cricothyroidotomy. Anaesthesia. 2008; 63: 535-539.

[49] Schaefer R, Hueter L, Preussler N, Schreiber T, Schwarzkopf K. Percutaneous transtracheal emergency ventilation with a self-made device in an animal model. Paediatric Anaesthesia. 2007; 17: 972-976.

[50] Price RJ, Laird C. A survey of surgical airway experiences and equipment among immediate care doctors. Emergency Medicine Journal. 2009; 26 : 438-441.

[51] Metterlein T, Frommer M, Ginzkey C, Becher J, Schuster F, Roewer N, et al. A randomized trial comparing two cuffed emergency cricothyrotomy devices using a wire-guided and a catheter-over-needle technique. The Journal of Emergency Medicine. 2011; 41: 326-332.

[52] Wong DT, Mehta A, Tam AD, Yau B, Wong J. A survey of Canadian anesthesiologists' preferences in difficult intubation and "cannot intubate, cannot ventilate" situations. Canadian Journal of Anesthesia. 2014; 61: 717-726.

[53] American College of Surgeons Commitee on Trauma. Advanced Trauma Life Support Program for Doctors. 9th edn. American College of Surgeons. 2012.

[54] Fennessy P, Drew T, Husarova V, Duggan M, McCaul CL. Emergency cricothyroidotomy: an observational study to estimate optimal incision position and length. British Journal of Anaesthesia. 2019; 122: 263-268.

[55] Wang EE, Quinones J, Fitch MT, Dooley-Hash S, Griswold-Theodorson $\mathrm{S}$, Medzon $\mathrm{R}$, et al. Developing technical expertise in emergency medicine-the role of simulation in procedural skill acquisition. Academic Emergency Medicine. 2008; 15: 1046-1057.

[56] Chan TC, Vilke GM, Bramwell KJ, Davis DP, Hamilton RS, Rosen P. Comparison of wire-guided cricothyrotomy versus standard surgical cricothyrotomy technique. The Journal of Emergency Medicine. 1999; 17: 957-962.

[57] DiGiacomo JC, Angus LD, Gelfand BJ, Shaftan GW. Cricothyrotomy technique: standard versus the rapid four step technique. The Journal of Emergency Medicine. 1999; 17: 1071-1073.

[58] Eisenburger P, Laczika K, List M, Wilfing A, Losert H, Hofbauer R, et al. Comparison of conventional surgical versus Seldinger technique emergency cricothyrotomy performed by inexperienced clinicians. Anesthesiology. 2000; 92: 687-690.

[59] Hill C, Reardon R, Joing S, Falvey D, Miner J. Cricothyrotomy technique using gum elastic bougie is faster than standard technique: a study of emergency medicine residents and medical students in an animal lab. Academic Emergency Medicine. 2010; 17: 666-669.

[60] Holmes JF, Panacek EA, Sakles JC, Brofeldt BT. Comparison of 2 cricothyrotomy techniques: standard method versus rapid 4-step technique. Annals of Emergency Medicine. 1998; 32: 442-446.

[61] Johnson DR, Dunlap A, McFeeley P, Gaffney J, Busick B. Cricothyrotomy performed by prehospital personnel: a comparison of two techniques in a human cadaver model. The American Journal of Emergency Medicine. 1993; 11: 207-209.

[62] Keane M, Brinsfield K, Dyer K, Roy S, White D. A laboratory comparison of emergency percutaneous and surgical cricothyrotomy by prehospital personnel. Prehospital Emergency Care. 2004; 8: 424-426.
[63] Murphy C, Rooney SJ, Maharaj CH, Laffey JG, Harte BH. Comparison of three cuffed emergency percutaneous cricothyroidotomy devices to conventional surgical cricothyroidotomy in a porcine model. British Journal of Anaesthesia. 2011; 106: 57-64.

[64] Salah N, Mhuircheartaigh RN, Hayes N, McCaul C. A comparison of four techniques of emergency transcricoid oxygenation in a manikin. Anesthesia and Analgesia. 2010; 110: 1083-1085.

[65] Schaumann N, Lorenz V, Schellongowski P, Staudinger T, Locker GJ, Burgmann $\mathrm{H}$, et al. Evaluation of Seldinger technique emergency cricothyroidotomy versus standard surgical cricothyroidotomy in 200 cadavers. Anesthesiology. 2005; 102: 7-11.

[66] Schober P, Hegemann MC, Schwarte LA, Loer SA, Noetges P. Emergency cricothyrotomy-a comparative study of different techniques in human cadavers. Resuscitation. 2009; 80: 204-209.

[67] Sulaiman L, Tighe SQM, Nelson RA. Surgical vs wire-guided cricothyroidotomy: a randomised crossover study of cuffed and uncuffed tracheal tube insertion. Anaesthesia. 2006; 61: 565-570.

[68] Chua H, Wong T, Lim WY, Wong P. Emergency front-of-neck airway in the COVID-19 patient: Cannula or surgical cricothyroidotomy? Anaesthesia Critical Care \& Pain Medicine. 2020; 39: 475-477.

[69] Bribriesco A, Patterson GA. Cricothyroid approach for emergency access to the airway. Thoracic Surgery Clinics. 2018; 28: 435-440.

[70] Heard A, Gordon H, Douglas S, Grainger N, Avis H, Vlaskovsky P, et al. Front-of-neck airway rescue with impalpable anatomy during a simulated cannot intubate, cannot oxygenate scenario: scalpel-fingercannula versus scalpel-finger-bougie in a sheep model. British Journal of Anaesthesia. 2020; 125: 184-191.

[71] Umek N, Hodzovic I, Damjanovska M, Cvetko E, Zel J, Seliskar A, et al. Rescue oxygenation success by cannula or scalpel-bougie emergency front-of-neck access in an anaesthetised porcine model. PLoS ONE. 2020; 15: e0232510.

[72] Heymans F, Feigl G, Graber S, Courvoisier DS, Weber KM, Dulguerov P. Emergency cricothyrotomy performed by surgical airway-naive medical personnel: a randomized crossover study in cadavers comparing three commonly used techniques. Anesthesiology. 2016; 125: 295-303.

[73] Paix BR, Griggs WM. Emergency surgical cricothyroidotomy: 24 successful cases leading to a simple 'scalpel-finger-tube' method. Emergency Medicine Australasia. 2012; 24: 23-30.

[74] Hubble MW, Wilfong DA, Brown LH, Hertelendy A, Benner RW. A meta-analysis of prehospital airway control techniques part II: alternative airway devices and cricothyrotomy success rates. Prehospital Emergency Care. 2010; 14: 515-530.

[75] Wong DT, Prabhu AJ, Coloma M, Imasogie N, Chung FF. What is the minimum training required for successful cricothyroidotomy? A study in mannequins. Anesthesiology. 2003; 98: 349-353.

[76] Walls RM. Cricothyroidotomy. Emergency Medicine Clinics of North America. 1988; 6: 725-736.

[77] Joffe AM, Aziz MF, Posner KL, Duggan LV, Mincer SL, Domino KB. Management of difficult tracheal intubation. Anesthesiology. 2019; 131: 818-829.

[78] Bair AE, Panacek EA, Wisner DH, Bales R, Sakles JC. Cricothyrotomy: a 5 -year experience at one institution. The Journal of Emergency Medicine. 2003; 24: 151-156.

[79] American Society of Anesthesiologists Task Force on Management of the Difficult Airway. Practice guidelines for management of the difficult airway: an updated report by the American Society of Anesthesiologist Task Force on management of the difficult airway. Anesthesiology. 2003; 98: 1269-1277.

[80] Lockey D, Crewdson K, Weaver A, Davies G. Observational study of the success rates of intubation and failed intubation airway rescue techniques in 7256 attempted intubations of trauma patients by prehospital physicians. British Journal of Anaesthesia. 2014; 113: 220-225.

[81] Mabry RL. An analysis of battlefield cricothyrotomy in Iraq and Afghanistan. Journal of Special Operations Medicine. 2012; 12: 17-23.

[82] Pracy JP, Brennan L, Cook TM, Hartle AJ, Marks RJ, McGrath BA, et al. Surgical intervention during a can't Intubate can't Oxygenate (CICO) event: emergency Front-of-neck Airway (FONA)? Clinical Otolaryngology. 2016; 41: 624-626.

[83] Atkins JH, Rassekh CH. Multidisciplinary airway response teams: concept, structure \& implementation. Operative Techniques in 
Otolaryngology-Head and Neck Surgery. 2020; 31: 105-110.

[84] Mark L, Lester L, Cover R, Herzer K. A decade of difficult airway response team. Critical Care Clinics. 2018; 34: 239-251.

[85] Atkins JH, Rassekh CH, Chalian AA, Zhao J. An airway rapid response system: implementation and utilization in a large academic trauma center. Joint Commission Journal on Quality and Patient Safety. 2017; 43: 653660.

[86] Panda N, Donahue DM. Acute airway management. Annals of Cardiothoracic Surgery. 2018; 7: 266-272.
How to cite this article: Federico Mazza, Massimiliano Venturino, Davide Turello, Alberto Gorla, Cristina Degiovanni, Alessandro Locatelli, et al. Cricothyroidotomy in the emergency setting: indications, techniques and outcomes.

Signa Vitae. 2021;17(3):31-41. doi:10.22514/sv.2021.063. 